\title{
MUSIVARIA HD MUSEO VIRTUAL DE MOSAICOS DE LA HISPANIA ROMANA, GRÁFICOS Y HERRAMIENTAS PARA LA VISUALIZACIÓN DE DATOS SOBRE MOSAICOS ROMANOS TARDOANTIGUOS.
}

\author{
BEATRIZ GARRIDO RAMOS \\ UNED
}

Resumen

El presente artículo hace referencia a una serie de herramientas utilizadas para la visualización de datos de iconografía musivaria tardoantigua en la Península Ibérica. Nos centraremos en el Proyecto Musivaria HD, como resultado de la tesis doctoral. Posteriormente, en los gráficos, como principal forma de presentación de la información, y como ejemplo de visualización de datos, de manera que permite su análisis desde un punto de vista que difiere del habitual en las investigaciones sobre Historia del Arte en general, y mosaicos romanos tardoantiguos en particular. Dichas herramientas han permitido, en consecuencia, aproximarse a los datos desde la perspectiva de las Humanidades Digitales, lo que ha facilitado la extracción de conclusiones más enriquecedoras y a distintos niveles.

\section{Palabras clave}

Proyecto Musivaria HD, Humanidades Digitales, Base de datos, musivaria romana tardoantigua, Península Ibérica, Hispania, Visualización de datos, Open Access/Open Education.

\section{MUSIVARIA HD VIRTUAL MOSAIC MUSEUM OF ROMAN HISPANIA, GRAPHICS AND TOOLS FOR DISPLAYING DATA ON LATE ROMAN MOSAICS.}

\begin{abstract}
This article refers to a series of tools used for the visualization of late-ancient musivary iconography data in the Iberian Peninsula. We will focus on the Musivaria HD Project, as a result of the author's doctoral thesis. Subsequently, in graphs, as the main form of presentation of information, as an example of visualization of data, in a way that allows its analysis from a point of view that differs from that usual in investigations on the History of Art in general, and late Roman mosaics in particular. Consequently, these tools have made it possible to approach the data from the perspective of the Digital Humanities, which has facilitated the extraction of more enriching conclusions at different levels.
\end{abstract}

\section{Keywords}

Musivaria HD Project, Digital Humanities, Database, late Roman ancient musivaria, Iberian Peninsula, Hispania, Data Visualization, Open Access/Open Education. 


\section{Introducción}

El patrimonio cultural peninsular es elevado y de incalculable valor. Buen ejemplo de ello se encuentra en el ámbito de los mosaicos romanos tardoantiguos que es el área de estudio del presente trabajo. El gran volumen de datos existente relativo a la musivaria de la Península Ibérica en época romana condujo al diseño de una herramienta que permitiese aglutinar y gestionar de manera sencilla y eficaz toda la información, que se adaptara a determinados estándares para aumentar su utilidad, y cumpliera igualmente su cometido de difusión social y puesta en valor de un patrimonio cultural que con frecuencia no es lo suficientemente valorado.

La evolución histórico-artística que se sucedió en la Península Ibérica en época romana fue, probablemente y en gran medida, consecuencia del desarrollo de la cultura indígena anterior (cultos, deidades, etc.), que como es lógico, dejaría restos de su existencia, tanto en lo referente a cuestiones geográficas (evolución del paisaje) como en el arte en general y en la iconografía romana en particular.

A este respecto, y para contextualizar el contenido existente en la base de datos (en adelante $\mathrm{BD}$ ) realizada así como en el catálogo resultante, hay que exponer que los ejemplos iconográficos recopilados y estudiados proceden tanto de yacimientos, villae y domus, como de mosaicos aislados - cuya ubicación desconocemos-. De estos últimos se carece de suficiente información y, en consecuencia, no se tiene claro si pudieron situarse en una vivienda o en otro lugar.

\section{Aproximación metodológica para el estudio de los mosaicos romanos en Hispania}

El marco teórico-metodológico que tomaremos como punto de partida será el de las HD y el concepto que la autora ha denominado "Geografía del Mosaico" como nueva vía que pretende una aproximación a la iconografía desde el estudio de los vestigios arqueológicos atendiendo al análisis de la cultura material musivaria repartida por la geografía de la Península Ibérica en época romana.

Nuestra aproximación a la musivaria de época romana en Hispania se producirá desde la disciplina de la Historia del Arte - concretamente la Iconografía- y desde la Geografía, llevando a cabo el estudio iconográfico y mitológico de las representaciones musivas y su distribución geográfica en el territorio peninsular.

El ámbito de la investigación, como se expone, se circunscribe a la Hispania romana. Por el momento se han repertorizado un buen número de villas, pero la intención en un futuro es la de abarcar todas las villas tardías peninsulares, a diferencia de los trabajos realizados hasta el momento, los cuales se centraban en zonas geográficas específicas.

Por ello, una de las principales aportaciones "ha sido la recuperación de textos, y la unificación y clasificación de todo ese material existente hasta la fecha, destacando entre otros textos los Corpus de Mosaicos de España que conforman sin duda una importantísima fuente de conocimiento para toda persona interesada en el ámbito concreto de la musivaria. No obstante, además de los corpus, se han tomado como referencia los estudios de un buen número de autores como Blázquez, Lancha, Morand, Chavarría y Fernández Castro entre otros, que previamente habían realizado estudios sobre mosaicos en diversas zonas de España"1.

\section{Investigación de la iconografía musivaria romana desde la perspectiva de las} Humanidades Digitales y la filosofía Open Access

Hasta la fecha se han llevado a cabo diversos proyectos ${ }^{2}$ a nivel nacional e internacional relativos al ámbito de la musivaria. También existen recursos de difusión como el portal de cultura http://geo.spainisculture.com/index.php/es.

\footnotetext{
${ }^{1}$ Garrido, 2017a: 82-83.

${ }^{2}$ Fuentes o proyectos digitales que han trabajado el tema de la musivaria dentro y fuera de España, por citar algunos ejemplos, son:

$<$ http://www.asprom.org/resources/links.html>.

$<$ http://m.sussex.ac.uk/byzantine/mosaic/>.

<https://www.wga.hu/frames-e.html?/html/zgothic/mosaics/9/index.html>.
} 
El presente texto muestra el resultado de la investigación realizada para la tesis doctoral - defendida en la UNED de Madrid en noviembre de 2019- bajo el título "Iconografía musivaria en la Península Ibérica en época romana: investigación y difusión desde el campo de las Humanidades Digitales", calificada con Sobresaliente cum laude por su novedad y ámbito de aplicación de las Humanidades digitales (en adelante HD) al campo de la iconografía musivaria romana en Hispania.

Autores como Kirschenbaum ${ }^{3}$, Mc Carthy o Moretti ${ }^{4}$ entre otros, se han referido ampliamente al concepto de HD, pero no profundizaremos en ello puesto que no es el cometido de este estudio. En su lugar mencionaremos otro término destacable que es el de "cultura conectada", establecido previamente para mostrar la necesidad de "conectar", de una forma homogénea, accesible y estandarizada la información y los resultados de la investigación. Buena prueba de ello es el movimiento "Open Access/Open Education" cuya filosofía invita a un aperturismo sin duda necesario. En relación a ello, cabe destacar que este tipo de proyectos suponen un gran avance como divulgación de contenidos y como transferencia del conocimiento a la sociedad.

Es sumamente importante destacar el nuevo abanico de posibilidades que brinda este nuevo movimiento, al igual que sucede con la nueva aproximación metodológica desde las HD y las TIC, así como el uso de programas específicos para el tratamiento y visualización de la información y la realización de estadísticas. A este respecto, siguiendo a Rincón Zamorano "con el acceso a una mayor cantidad de información, tanto en cantidad como en variedad, surgen nuevos problemas para su tratamiento, siendo necesarios nuevos métodos y tecnologías. La informática se encarga de definir estos procedimientos y tecnologías que nos facilitan el manejo de grandes volúmenes de información de una manera amigable"7.

A colación de las palabras de Rincón Zamorano, a continuación, se describirá el proyecto Musivaria HD, sus principales características y su finalidad para el estudio y la investigación de la iconografía musivaria en Hispania.

\section{Proyecto Musivaria HD: Herramienta, BD y catálogo para los mosaicos romanos tardoantiguos}

El registro digital de la información obtenida durante el proceso de investigación se realizó en una web en la que se incorporaron e integraron los datos resultantes del estudio de las villas tardoantiguas tales como texto, fotografías, plantas de villas, planos, reconstrucciones 3D y vídeos, así como documentación en formato pdf para su posterior descarga. La estructura de la aplicación, desarrollada de forma integrada y modular, se implementa con herramientas open source.

El sistema fue diseñado siguiendo la Norma CIDOC-CRM ${ }^{8}$. El Modelo de Referencia Conceptual (CRM) se trata de una ontología formal aplicada en el ámbito del Patrimonio Cultural. Es llevada a cabo por un grupo de trabajo del Comité de Documentación que se desarrolla en el Consejo Internacional de Museos (ICOM) que el 12 de septiembre de 2006 hizo oficial la norma ISO 21127: 2006 ${ }^{9}$. En diciembre de 2014 se presentó una nueva versión basada en la versión 5.0.4 del CIDOC CRM, la ISO 21127: 2014. Cabe destacar que el Grupo

\footnotetext{
$<$ https://www.archesproject.org/what-is-arches/>.

$<$ http://www.seemosaics.org/>.

3 Véase: <https://mkirschenbaum.files.wordpress.com/2011/03/ade-final.pdf>.

4 Autor que estudia grandes corpus de textos y se refiere en su discurso a conceptos como el de la cuantificación, visualización y la lectura distante (Distant Reading).

${ }^{5}$ Garrido-Ramos, 2017a: 12.

${ }^{6}$ La propuesta que se realiza se trata de una base de datos en Acceso Abierto, lo que está más cerca de un recurso educacional abierto. Para ampliar información sobre el concepto Open Access/Open Education véase: $<$ https://sparcopen.org/>

7 Rincón, 2015: 2.

8 "Working Groups". En: <http://network.icom.museum/cidoc/working-groups/overview/>. [01/07/2017].

9 Para ampliar información sobre la norma ISO 21127:2006 véase "Information and documentation -A reference ontology for the interchange of cultural heritage information". En: <https://www.iso.org/standard/34424.html> [15/06/2017].
} 
de Trabajo de Normas para la Documentación del CIDOC ha desarrollado una declaración de principios de documentación para los museos ${ }^{10}$, y además, trabaja en diversas cuestiones entre las que se encuentra el fomento de vocabularios controlados y recursos terminológicos específicos de relevancia para museos, como es el caso de Iconclass $^{11}$, clasificación internacional que se ha aplicado a los mosaicos que conforman nuestra BD.

El proyecto Musivaria $H D^{12}$ es una web única y pionera en su campo por albergar un catálogo de mosaicos (procedentes de una base de datos llevada a cabo para la investigación de la tesis doctoral), cuestión importante sin duda si se tiene en cuenta que habitualmente no se encuentran concentrados de esta forma, y menos online, principalmente porque suele recurrirse a su estudio de forma aislada, por zonas o yacimientos, no en conjunto como se ha realizado en este caso. La presentación de la información de esta forma permite una nueva visualización que facilita la valoración e interpretación de los datos y estadísticas en su conjunto, contribuyendo a la formulación de nuevas preguntas de investigación. A este respecto podemos mencionar a modo de ejemplo los resultados obtenidos de la aplicación del método cualitativo de triangulación de datos para la zona de Cataluña y Levante, lo que ha permitido proponer un posible taller de motivos bicromos caracterizados por la torpeza en la ejecución por parte del artista ${ }^{13}$.

La web dispone de un menú principal en la cabecera, con sus correspondientes apartados (pestañas) y subapartados, de manera que facilita la navegación del usuario y le presenta de forma intuitiva y rápida toda la información existente en la página.

Entre otros aspectos cabe exponer que combina las tecnologías PHP, HTML5, CSS3 y JavaScript con el Diseño Web Adaptativo (Responsive Web Design) destinado a su visualización en cualquier dispositivo (smartphone, tablet, ordenadores portátiles, etc.).

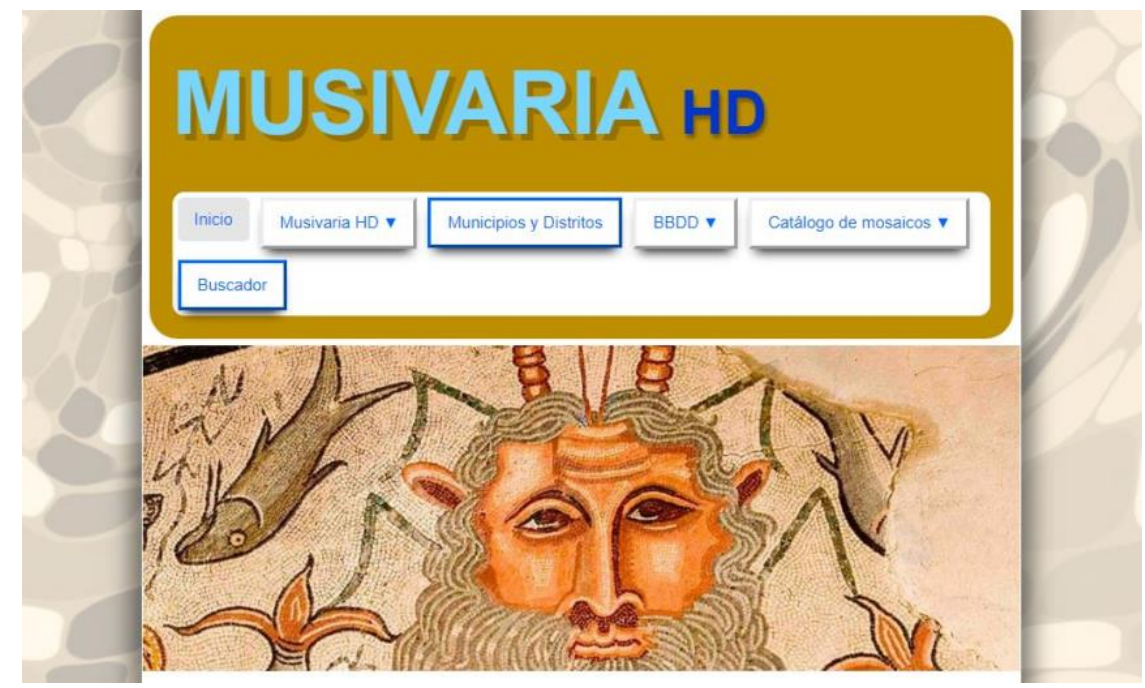

Fig. 1. Cabecera y menú principal de la web Musivaria HD.

10 Tal y como se expone en la web del CIDOC: "El grupo de trabajo tiene como objetivo actuar como un punto de enlace con los órganos e iniciativas relevantes que desarrollan las normas de relevancia para la documentación de los museos. Ejemplos de tales cuerpos son el Text Encoding Initiative, CCO y MICHAEL. Los miembros del grupo están trabajando en una asignación de TEI a la CIDOC CRM”.

${ }^{11}$ Es una buena alternativa para trabajar con imágenes porque permite gestionar vocabularios controlados para la descripción o clasificación de iconos o figuras: <http://www.iconclass.nl/home >. Otros ejemplos son Getty Research Institute <http://www.getty.edu/research/tools/vocabularies/aat/>, Pelagios $<$ http://pelagiosproject.blogspot.com.es/>, Ariadne <http://www.ariadneinfrastructure.eu/> y Perseus $<$ http://www.perseus.tufts.edu/hopper/>. (Garrido, 2017c: 21).

${ }_{12}$ Disponible en línea: <https://musivariahd.com/>.

${ }^{13}$ En la web se cita el caso procedente de Pujol de Benicató. 
A continuación, se explicará brevemente la funcionalidad de cada uno de los menús de la web. Para empezar, en el menú de cabecera tendremos las siguientes pestañas:

○ Inicio.

- Musivaria HD: en el que se encuentran los siguientes apartados:

- Proyecto Musivaria HD: breve descripción sobre el proyecto.

- Gráficos de Musivaria HD: distintas estadísticas que permitirán analizar la información existente en la BD.

- Gráficos de TaxMOS HD ${ }^{14}$ : estadísticas sobre los términos utilizados en la taxonomía (Fig. 3) creada durante la tesis a la que se puede acceder directamente desde la página principal.

- Geografía del mosaico ${ }^{15}$ : recopilación de distintos mapas que muestran brevemente el recorrido por la metodología utilizada en la investigación y el estudio de la musivaria romana desde la perspectiva de la geografía.

- Bibliografía: consulta de las referencias que se han utilizado para la realización de la BD sobre mosaicos.

- Contacto: para que los usuarios puedan dirigirse a la autora con la intención de realizar mejoras en la web.

- Municipios (España) y Distritos (Portugal) ${ }^{16}$ : acceso directo a los distintos municipios y distritos de conforman el catálogo de mosaicos. A través de enlaces (hipertexto) se podrá acceder a los mosaicos existentes en cada lugar.

- $B D$ : para consultar de forma rápida y sencilla los mosaicos objeto de estudio de manera que el usuario pueda seleccionar los existentes en cada lugar, ya sea un yacimiento, una villa de España, una villa de Portugal, una domus de España, una domus de Portugal o un lugar indeterminado (refiriéndonos por indeterminado a los casos de los que se carece de suficiente información para establecer una clasificación concreta del lugar) y otros casos.

- Catálogo de mosaicos: permite acceder directamente a cada uno de los mosaicos repertorizados para su consulta.

○ Buscador $r^{17}$ : permite al usuario realizar dos tipos de consultas, sencilla y avanzada. Nos referiremos a dicho apartado posteriormente con más detenimiento.

Ahora nos detendremos en la última pestaña del menú principal que es la relativa al buscador. Gracias al motor de búsqueda interno el usuario podrá efectuar dos tipos de búsqueda: sencilla o avanzada. Los criterios y sugerencias de búsqueda son los siguientes:

o Búsqueda sencilla: consulta de término/s de cualquier campo de manera que se recuperará información existente en toda la web y en la BD de Musivaria HD.

○ Criterios según:

- País: España (municipios) o Portugal (distritos).

- Lugar: yacimiento, villa, domus, indeterminado, otros casos.

${ }^{14}$ Disponible en línea: <https://musivariahd.com/graficos-de-taxmos-hd.html>. Este apartado se refiere a los gráficos resultantes del vocabulario realizado para la investigación y que se encuentra disponible para su consulta en línea: <https://www.vocabularyserver.com/taxmos/index.php>. Tal y como se expone en la propia web del vocabulario TaxMOS HD - Taxonomía para la investigación y difusión de la iconografía musivaria romana desde las Humanidades Digitales: "Esta taxonomía sobre la investigación y difusión de la iconografía musivaria en la Hispania romana desde la perspectiva de las Humanidades Digitales ha sido desarrollada para intentar subsanar la falta de unificación de la información relativa a esa rama de la Historia del Arte que se encuentra con frecuencia dispersa y sin estandarizar, con el fin de que pueda ser utilizada por historiadores e investigadores para llevar a cabo estudios sobre la misma, así como por la comunidad de bumanistas digitales a la que puede resultar de interés por tratarse de un ejemplo de aplicación de las HD a la Historia del Arte. La taxonomía tiene como principal objetivo difundir la cultura material que conforma la musivaria romana tardoantigua de la Península Ibérica, asi como estructurar y difundir a su vez. la información referente a las Humanidades Digitales y a la iconografía musivaria, tema sobre el que versa la tesis doctoral de la autora. Se espera que la taxonomía sea particularmente útil para el análisis de la información sobre iconografía y mitología romanas, las principales técnicas constructivas en Hispania y el tipo de decoración de sus mosaicos, asi como las herramientas, métodos y estándares aplicados a la Historia del Arte desde el campo de las Humanidades Digitales".

15 Para ampliar información en relación al concepto propuesto por la autora véase: $<$ https://musivariahd.com/geografia-del-mosaico-y-triangulacion-de-datos.html $>$.

16 Véase: https://musivariahd.com/municipios-y-distritos-de-la-bbdd.html.

17 Véase: <https://musivariahd.com/buscador.html>. 
- Mosaico: podremos a su vez realizar búsquedas relativas a:

- nombre de mosaico: en base al catálogo que se adjunta en la web.

- tipo de mosaico: geométrico o figurativo.

- tema de mosaico: acuático, agrícola, alimento/culinario, arquitectura, astronomía, cacería, calzado, circo, epigrafía, fauna, figura humana, flora, geometría, lucha, marino, mitología, naturaleza, objeto, solar, vegetal.

- Palabras clave o descriptores (metadatos por los que se clasificaron previamente los mosaicos repertorizados): $739^{18}$ en total. El usuario puede visualizarlos mediante el correspondiente listado de palabras que se le presenta para facilitar la tarea de búsqueda según interés de una determinada palabra.

Otra de las características de interés en la web es la posibilidad de navegar entre contenido enlazado mediante hipertexto, es decir, entre contenido que guarde estrecha relación entre sí, de manera que contribuya a una experiencia diferente para cada usuario según el lugar sobre el que desee ampliar información.

Por otra parte, en el menú principal también se dispone de la pestaña referente al contacto, que confiamos contribuirá a la mejora del contenido existente en la web, ya sea mediante la inserción de nuevos mosaicos, mapas, vídeos, gráficos o estadísticas procedentes del material que los usuarios puedan hacer llegar a la autora. Esto permitirá analizar el grado de interés por el tema de la musivaria en general, y de posibles casos en particular, de manera que facilite la extracción de conclusiones al respecto de la navegación, la funcionalidad y utilidad de la información que se ha puesto a disposición de la comunidad científica y de toda persona interesada en ámbito de la musivaria romana en Hispania.

Finalmente, la herramienta resulta totalmente interactiva, y a ello contribuye sin duda el apartado de vídeos con reconstrucciones y modelos en 3D de algunos lugares estudiados durante la investigación y la repertorización de los mosaicos. Todo este material permitirá al usuario hacerse una idea del tipo de espacio y de los mosaicos a los que nos estamos refiriendo, gracias a la "visita virtual" del lugar. Este es solo un ejemplo de los recursos por los que podrá navegar el usuario de una forma libre y sencilla.

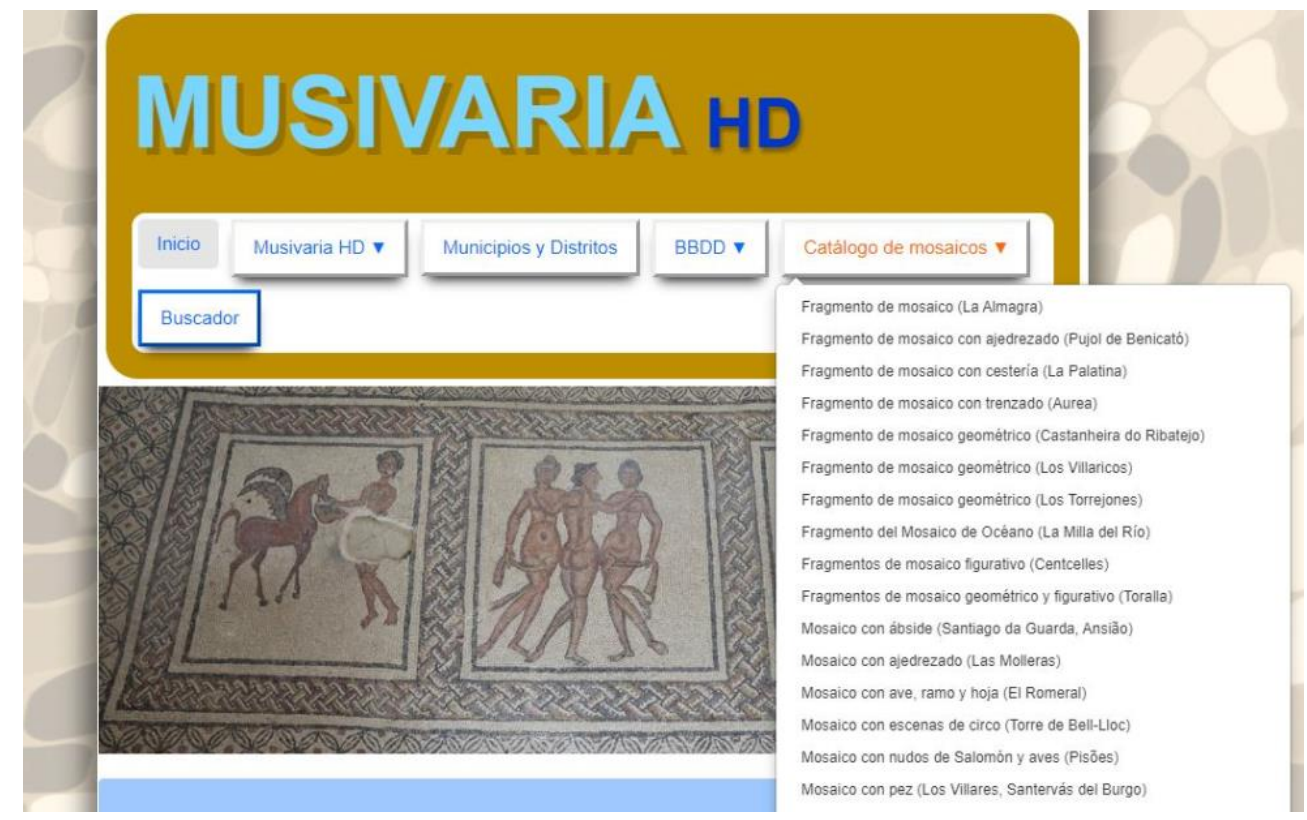

Fig. 2. Catálogo que muestra una parte de los mosaicos repertorizados en la BD.

18 Véase: <https://musivariahd.com/listado-de-descriptores-o-palabras-clave.html>. 
- vocabularyserver.com/taxmos/index.php?tema $=378 /$ mosaicos-romanos

\section{TaxMOS HD - Taxonomía para la investigación y difusión de la iconografía musivaria romana desde las Humanidades Digitales

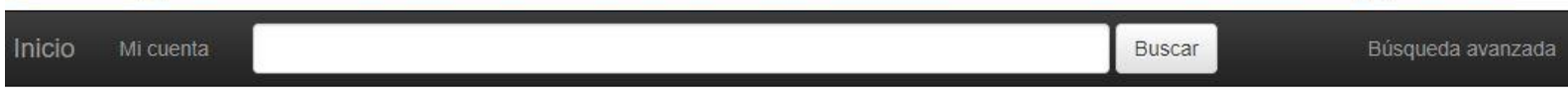

\section{mosaicos romanos}

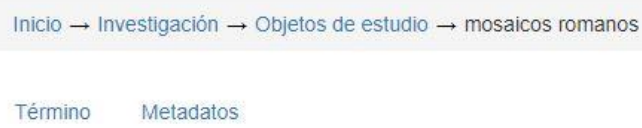

Fig. 3. Taxonomía TaxMOS HD. Ejemplo de término "mosaicos romanos": https://www.vocabularyserver.com/taxmos/index.php?tema=37\&/mosaicos-romanos

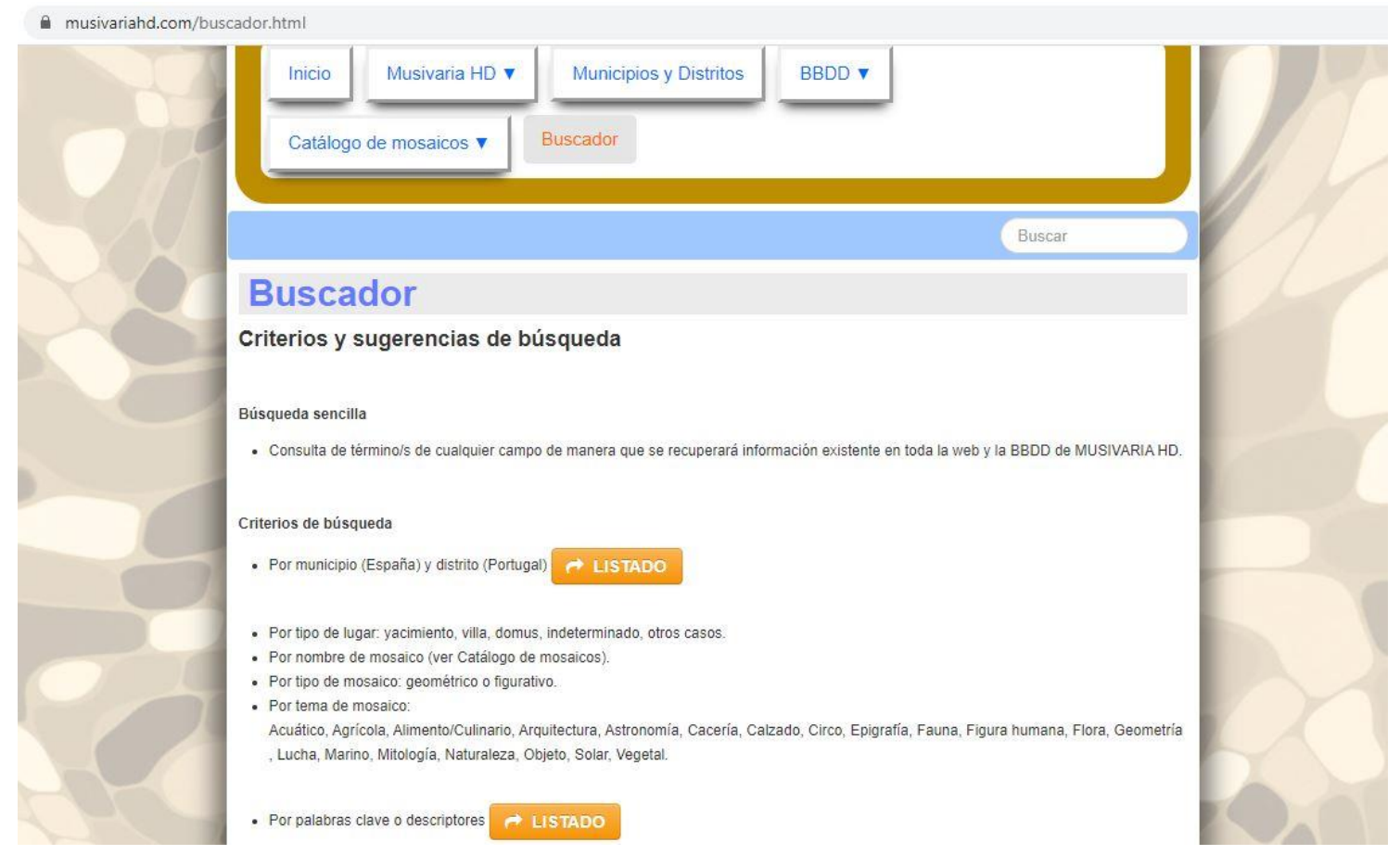

Fig. 4. Buscador que muestra los criterios y sugerencias de búsqueda. 
A continuación, a modo de resumen, se muestra una tabla con las principales características de Musivaria HD en cuanto a contenido y forma de presentación de la información.

\begin{tabular}{|c|c|c|}
\hline $\begin{array}{c}\text { Musivaria } \\
\text { HD }\end{array}$ & $\begin{array}{l}\text { CARACTERÍSTICAS Y } \\
\text { TIPO DE INFORMACIÓN } \\
\text { - Web sobre musivaria } \\
\text { romana que reúne toda } \\
\text { la información recopilada } \\
\text { durante la investigación } \\
\text { así como los principales } \\
\text { resultados obtenidos. } \\
\text { - Catálogo de mosaicos } \\
\text { tardoantiguos en la Península } \\
\text { Ibérica. } \\
\text { - Bibliografía de diverso tipo } \\
\text { para profundizar en el estudio } \\
\text { de la musivaria romana en } \\
\text { Hispania. }\end{array}$ & $\begin{array}{l}\text { PRESENTACIÓN } \\
\text { DE LA INFORMACIÓN } \\
\square \text { Menús navegables } \\
\square \text { Contenido enlazado } \\
\text { (hipertexto) } \\
\square \text { Gráficos } \\
\square \text { Mapas (geolocalización) } \\
\square \text { Imágenes } \\
\square \text { Reconstrucciones 3D } \\
\square \text { Vídeos }\end{array}$ \\
\hline
\end{tabular}

Tabla 1. Principales características e información de Musivaria HD.

\section{Una forma de presentación de la información: los gráficos}

A colación de la utilización de gráficos para presentar la información en la web Musivaria $\mathrm{HD}$, hemos considerado necesario realizar un breve apunte sobre dicha cuestión. Franco Moretti al hablar de un "sistema colectivo", de un todo, refiriéndose por "todo" a lo que él denomina "artefactos o artificios", que serían tres, los mapas, los gráficos y los árboles, nos hace reflexionar sobre el conjunto de datos (dataset), que en ningún caso deben tratarse de forma aislada, porque carecería de sentido que así fuera. En nuestra investigación, además de analizar de forma individual los registros, hemos recurrido a la visualización de datos de una forma "colectiva", en su conjunto, con el fin de intentar extraer un mayor número de conclusiones, y además, se ha procedido a la geolocalización de la información mediante mapas y la presentación de resultados con gráficos, tal y como expone Moretti.

Teniendo en cuenta la opinión de Moretti, y retomando la cuestión gráfica, en primer lugar, a la hora de diseñar una representación visual, se deben tener claros los objetivos que se persiguen, ya que es algo a tener muy presente para poder expresar la información con totales garantías de que permita extraer el máximo partido de la misma ${ }^{19}$.

Una vez recopilada y analizada toda la información necesaria para llevar a cabo un trabajo o proyecto tanto en Humanidades como en cualquier otro campo, y mostrar los resultados obtenidos o la información seleccionada y estudiada, se torna necesario poder representarla. Para llevar a cabo dicha tarea, se podría utilizar todo tipo de gráficos, pero los más fáciles de comprender por parte de los usuarios son los bidimensionales (2D) y tridimensionales (3D), que permiten en un golpe de vista rápido captar una gran cantidad de información. En el caso del diseño bidimensional, se suele recurrir a él porque presenta la información de forma clara y directa, siendo la manera más fácil de asimilar la información por parte de la persona que la visualice. Con el color o una forma simple podremos transmitir un mensaje conciso al espectador.

Por otra parte, el diseño tridimensional es más realista, y en general, la tendencia actual apunta a su mayor utilización, como ha sucedido en los últimos años (cuestión que ha quedado patente en diversos campos como el de la realidad virtual, la fotografía, el diseño, la ilustración, etc.). En definitiva, "se puede optar por el gráfico 2D cuando la intención sea

\footnotetext{
${ }^{19}$ Garrido-Ramos, 2017c: 17-18.
} 
la de centrar la atención en la información que se presente, y por el 3D, cuando se prefiera presentar dicha información de una forma más ilustrativa y realista" 20 .

No obstante, para ampliar información al respecto de los colores, la psicología de la forma y otras cuestiones relativas a la presentación de esa información se recomienda acudir a la denominada Teoría de la Gestalt (también conocida por "psicología de la forma" o "de la configuración") en la que se hace referencia a principios (Wertheimer, 1923) como el de la simplicidad y el de la relación figura-fondo. Los cinco principios básicos de la Gestalt son: proximidad (los elementos próximos se agrupan fácilmente), similitud (los elementos similares permiten su visualización grupal), juego de objetivos (agrupaciones de elementos afectados por una secuencia precedente), buena continuación (el campo conceptual se organiza en líneas de flujo ininterrumpido) y cierre (siempre que sea posible se agrupará los elementos para formar unidades).

En nuestro caso, y teniendo en cuenta que la presentación de un volumen importante de datos puede resultar difícil de comprender, recurriremos a unos gráficos sencillos para mostrar las estadísticas finales, en contraposición de la forma de presentar los datos por ejemplo en un programa que trabaje con grafos, y que como es lógico, debido a la relación entre los datos que están enlazados presenta un nivel de complejidad mayor que nada tiene que ver con un simple gráfico. Es importante recordar que a mayor sencillez gráfica mayor comprensión de la información por parte del usuario.

También conviene replantearse lo siguiente: ¿Qué es más importante, el contenido o la estética a la hora de presentar la información? ambas cuestiones son importantes, pero es lógico pensar que la visualización es algo fundamental. De poco servirá aglutinar y concentrar la información, todo el conjunto de datos, si a posteriori no fuéramos capaces de transmitirla, organizarla y presentarla convenientemente ${ }^{21}$.

Igualmente, se debería dar respuesta a otra pregunta, ¿qué queremos representar? En palabras de Rincón Zamorano, dicha pregunta se responde como sigue: "Utilizando la teoría de sistemas, que trata de conciliar las dos aproximaciones a la descripción de la realidad que existen: la analítica, basada en operaciones de reducción, y la sistémica, basada en operaciones de composición. En la teoría de sistemas se trata de describir todos los objetos y relaciones que existen en un determinado escenario, de manera que si simuláramos el comportamiento de estos objetos (como si fuera un mundo virtual) pudiéramos obtener el mismo resultado (con el margen de error que consideremos admisible) que el que obtendríamos en la realidad"22.

Para finalizar, debemos destacar la importancia que a nuestro modo de entender tiene la presentación de la información de una forma sencilla, en lo que entendemos juega un importante papel la visualización de datos, y en consecuencia, una correcta selección de las herramientas para llevarla a cabo, que en parte es lo que hemos querido transmitir mediante el uso de estas herramientas.

\section{Conclusión}

La combinación de una metodología cuantitativa y cualitativa en el estudio de la musivaria tardoantigua en Hispania ha devuelto unas conclusiones enriquecedoras. Recordemos que, tal y como se expone en la web Musivaria HD, la Geografía del Mosaico ha permitido proponer un posible taller itinerante cuyos artistas habrían trabajado en la zona de Cataluña y Levante, que se caracterizaría por la realización de obras bicromas en las que predomina una clara torpeza ejecutiva, aspecto que ha servido para su clasificación.

En cuanto a la aproximación desde las HD, como transformación del paradigma de la actividad científica por medio de la accesibilidad y el tratamiento de la información desde otra perspectiva, y retomando la idea del distant reading (concepto mencionado con anterioridad) de Moretti, por la que este autor cuantifica la literatura mundial, y el análisis

\footnotetext{
${ }^{20}$ Garrido-Ramos, 2017c: 18.

${ }^{21}$ Garrido-Ramos, 2017c: 20.

22 Rincón Zamorano, 2015: 2.
} 
macroscópico ${ }^{23}$ de Burello ${ }^{24}$ que se centra de forma exhaustiva ${ }^{25}$ en el objeto de estudio, debemos exponer que en nuestro corpus sobre iconografía musivaria romana, el lugar donde se ubica la obra musiva (tanto a nivel geográfico como de espacio arquitectónico) y el tipo de iconografía representada, son los parámetros más importantes y destacados.

Es decir, la visualización de datos con gráficos ha permitido extraer conclusiones del fenómeno de la musivaria contribuyendo a examinar los datos en su totalidad, analizando al máximo su contenido y dejando a un lado cuestiones subjetivas que pudieran sesgar o influir en el resultado. De esta forma cobra especial significado el análisis de la máquina frente al análisis humano.

Corroborando la idea de Moretti en lo que respecta a la lectura en la distancia, en nuestro caso se confirma que no es necesario proceder a la lectura global de toda la bibliografía en relación a un tema, por ejemplo, sobre musivaria en el siglo pasado y hasta la fecha, ya que es imposible que así sea. Pero si se analizan los datos desde otros puntos de vista es posible extraer conclusiones igualmente consistentes y fundamentadas como si hubiésemos procedido a una lectura cercana y profunda, a lo que Moretti denomina close reading. $\mathrm{Y}$ en este punto es donde cobra especial interés el uso de herramientas para la visualización de datos.

A este respecto se puede concluir que la visualización de datos en gráficos resulta de gran utilidad a la hora de realizar un análisis visual rápido de la información, puesto que facilita la comprensión de los datos. Además, proporciona estadísticas y porcentajes de forma automática, lo que permite hacer una correcta lectura de la información y ayudan a la comprensión de la misma. Por lo que respecta a la interfaz de usuario, resulta muy intuitiva y permite trabajar con cierta autonomía, sin necesidad de tener unos conocimientos excesivamente avanzados de la herramienta, siempre y cuando el volumen de datos con el que se trabaje no sea elevado y el tipo de relación entre nodos no resulte demasiado compleja.

Por tanto, teniendo presente lo expuesto anteriormente, la utilización de gráficos y la creación de la web Musivaria HD para la investigación y difusión de nuestro corpus de mosaicos, es un claro ejemplo de aproximación a la iconografía romana alejada por completo del análisis tradicional que suele llevarse a cabo, puesto que conlleva una forma de visualizar y analizar la información que dista por completo de la habitual. Es decir, "leer" la iconografía musivaria -mediante la aplicación de hipertexto en el caso de Musivaria HD-partiendo de un corpus seleccionado previamente y someterlo a estudio mediante la utilización de distintas herramientas que conduzcan a la formulación de nuevas preguntas de investigación y al planteamiento de hipótesis diferentes y novedosas, algo que no se ha realizado hasta la fecha en este ámbito.

\footnotetext{
${ }^{23}$ Vinculado al concepto de distant reading de Moretti. Véase Franco Moretti, Distant Reading. New York: VersoBooks, 2013.

${ }^{24}$ Burello, 2016: 582-584.

25 A este respecto Rodríguez Ortega alude a un "cambio de perspectiva -de escala- hacia lo global y exhaustivo". (Rodríguez Ortega, 2018: 1474-1487). Se pasaría por tanto del hasta ahora denominado por Moretti close reading (lectura directa) al distant reading (lectura distante), conceptos que ya han sido citados con anterioridad. Rodríguez Ortega se refiere precisamente al distant reading exponiendo que "implica el análisis de los fenómenos culturales en toda su posible exhaustividad” (Rodríguez Ortega, 2018: 1456).
} 


\section{Bibliografía}

Burello, M.: "Sobre Lectura distante, de Franco Moretti". En: Exlibris, 5, (2016), pp. 582584. En: $\quad$ Lhttp://revistas.filo.uba.ar/index.php/exlibris/article/view/3053> [02/06/2017].

Garrido Ramos, B.: "Mosaicos en la Hispania Romana y Humanidades Digitales". En: II

Congreso Internacional de Humanidades Digitales Hispánicas: Innovación, globalización e impacto, UNED, Madrid, 5-7 de octubre. (2015). En: <http://espacio.uned.es/congresosuned/index.php/hdh2015/hdh2015/paper/view/1 32> [01/08/2015].

Garrido-Ramos, B.: "Propuesta de base de datos y catálogo de mosaicos tardoantiguos en la Hispania romana". En: UCOARTE Revista de Teoría e Historia del Arte, 6, (2017a), pp. 924. En: <https://doi.org/10.21071/ucoarte.v6i.11001> [09/10/2018].

Garrido-Ramos, B.: TaxMOS HD - Taxonomía para la investigación y difusión de la iconografia musivaria romana desde las Humanidades Digitales. (2017b). En: $<$ https://vocabularyserver.com/taxmos/> [25/04/2018].

Garrido-Ramos, B.: "Visualización de datos y clasificación Iconclass: un estudio de caso desde la perspectiva de las Humanidades Digitales". En: Caracteres. Estudios culturales y críticos de la esfera digital, 6, 1, (2017c), pp. 10-33. En: <http://revistacaracteres.net/revista/vol6n1 mayo2017/iconclass/> [05/06/2018].

Garrido-Ramos, B.: Iconografía musivaria en la Peninsula Ibérica en época romana: investigación y difusión desde el campo de las Humanidades Digitales. Tesis Doctoral, UNED, Escuela Internacional de Doctorado (EIDUNED), Madrid, Programa de Doctorado en Historia e Historia del Arte y Territorio, 2019. En: <http://espacio.uned.es/fez/view/tesisuned:ED-Pg-HHAT-Bgarrido> [04/11/2019].

Moretti, F.: Distant Reading. New York: VersoBooks, 2013.

Proyecto Musivaria HD: Musivaria HD: <https://musivariahd.com/> [05/06/2019].

Rincón Zamorano, M.: “Representación formal de la información”. En: Curso de Experto Profesional en Humanidades Digitales, UNED, 2 (2015), p. 2.

Rodríguez Ortega, N.: "Ecosistema y explosión de las fuentes en la sociedad datacéntrica: nuevos materiales para la investigación cultural. Análisis del sistema expositivo español como estudio de caso". En: La formación artística: Creadores, historiadores, espectadores, XXI Congreso del Comité Español de Historia del Arte, editado por Ruiz, A. et al., 1474-1487. Santander: Editorial de la Universidad de Cantabria, 2018. 